\title{
Platelet-to-lymphocyte ratio: a prognostic factor for patients with advanced hepatocellular carcinoma?
}

\author{
Xiao-cai Tian • Fu-ren Zeng • De-hua Wu
}

Received: 17 December 2014 / Accepted: 26 January 2015 /Published online: 30 May 2015

(C) International Society of Oncology and BioMarkers (ISOBM) 2015

Dear Editor,

In a recent issue of Tumor Biology, Xing Li and his colleagues published an article [1] entitled "Platelet-tolymphocyte ratio acts as a prognostic factor for patients with advanced hepatocellular carcinoma." In this study, the investigators aimed to determine the prognostic value of platelet-to-lymphocyte ratio in a total of 243 advanced hepatocellular carcinoma (HCC) patients from two major hospitals. The investigators concluded that patients with a high platelet-to-lymphocyte ratio (PLR) had a lower 3-month survival rate (37.6 vs. $57.6 \%$ ) compared with patients with a low PLR. PLR was associated with aggressive malignant behavior and was not associated with the CLIP score and Child-Pugh grade. PLR was identified as an independent prognostic factor for advanced HCC patients not receiving systemic sorafenib. We would like to thank the authors for their contribution. However, we have several opinions that we would like to raise to the investigators.

Firstly, we suggest that the investigators should minimize the selection bias. PLR has been recently suggested to be a marker of thrombotic and inflammatory condition. In the literature, it was shown that valvular heart diseases, acute coronary syndromes, hypertension, end-stage renal diseases, and other diseases may potentially affect the PLR [2-6]. In the study of Xing Li et al. [1], patients with previous and/or

X.-c. Tian • D.-h. Wu $(\bowtie)$

Department of Radiation Oncology, Nanfang Hospital, Southern Medical University, Guangzhou 510515, People's Republic China e-mail: wudehua2014@126.com

X.-c. Tian · F.-r. Zeng

Department of Oncology, Hunan Province Geriatric Hospital, Changsha, Hunan 410016, People's Republic China secondary cancers were excluded, while such as heart diseases which is very common in old people (age 57 (19-86)) were not mentioned. Thus, it would be more relevant if the investigators had mentioned these PLR-affecting factors while evaluating PLR as an independent prognostic factor for advanced HCC patients.

Secondly, PLR may be altered by many medications such as immunosuppressive drug, steroid, antibiotic, and so on. In their study, 10 patients had received chemotherapy. It would have been better if the authors indicated clearly when the blood samples were taken or the time between taking the blood samples and giving chemotherapy.

Thirdly, the investigators just collected patients from two hospitals in the same city. The selected 243 patients were not enough to be the representative of all advanced hepatocellular carcinoma patients. More centers and more patients from different areas should be taken in if the authors want to identify PLR as an independent prognostic factor in advanced HCC patients.

Conflicts of interest None

\section{References}

1. Li X, Chen ZH, Xing YF, Wang TT, Wu DH, Wen JY, Chen J, Lin Q, Dong M, Wei L, Ruan DY, Lin ZX, Wu XY, Ma XK. Platelet-tolymphocyte ratio acts as a prognostic factor for patients with advanced hepatocellular carcinoma. Tumour Biol. 2014;36(4):2263-9. doi:10. 1007/s13277-014-2833-9.

2. Akkaya E, Gul M, Ugur M. Platelet to lymphocyte ratio: a simple and valuable prognostic marker for acute coronary syndrome. Int J Cardiol. 2014;177:597-8.

3. Oylumlu M, Yildiz A, Oylumlu M, Yuksel M, Polat N, Bilik MZ, Akyuz A, Aydin M, Acet H, Soydinc S. Platelet-to-lymphocyte ratio is a predictor of in-hospital mortality patients with acute coronary 
syndrome. Anatol J Cardiol. 2015;15(4):277-83. doi:10.5152/akd. 2014.5366.

4. Sarikaya M, Dogan Z, Ergul B, Filik L. Platelet-to-lymphocyte ratio for early diagnosis of celiac disease. Indian J Gastroenterol. 2015;34(2):182-3.

5. Sunbul M, Gerin F, Durmus E, Kivrak T, Sari I, Tigen K, et al. Neutrophil to lymphocyte and platelet to lymphocyte ratio in patients with dipper versus non-dipper hypertension. Clin Exp Hypertens (New York, NY : 1993). 2014;36:217-21. doi:10.3109/10641963. 2013.804547.

6. Turkmen K, Erdur FM, Ozcicek F, Ozcicek A, Akbas EM, Ozbicer A, et al. Platelet-to-lymphocyte ratio better predicts inflammation than neutrophil-to-lymphocyte ratio in end-stage renal disease patients. Hemodial Int. 2013;17:391-6. 